\title{
Impact of Membrane Resistance on Width and Amplitude of Spikes in Different Injected Currents in One Spiking Neural Model
}

\author{
SHIMA PILEHVARI, LEI ZHANG, RENE V. MAYORGA \\ Faculty of Engineering and Applied Science \\ University of Regina \\ 3737 Wascana Parkway, Regina Saskatchewan, S4S 0A2
}

CANADA

\begin{abstract}
Neurons in the brain as the elementary processing units and nervous system play a key role. If a neuron gets a proper stimulus, it produces action potentials (spikes) that are transferred along its axon. Reaching the end of the neuron, other neurons or muscle cells may be activated [1].

The effect of neural morphology along with thickness of dendrites and passive electrical parameters on the spikes width and amplitude can be investigated by analytical and numerical investigations of spiking models. The impact of mentioned proper stimulus may be degraded by passing time.

In this paper, it is tried to add the effective parameter 'membrane resistance' in well-known Hodgkin-Huxley model with four dimensions to compare several outputs due to changing resistance and various injected current. The goal of this paper has been to measure spikes changes or even how to determine current threshold when resistance is not constant (non-linear time dependant) result of different factors.
\end{abstract}

Key-Words: Spikes, Hodgkin-Huxley Model, Injected Current Electrical Parameters, Membrane Resistance, Amplitude and Width, Current Threshold, Izhikevich Model.

Received: February 18, 2021. Revised: July 5, 2021. Accepted: July 18, 2021. Published: July 26, 2021.

\section{Introduction}

Neurons in the human brain (86 billions (Azevedo et al., 2009)) is approximately six times the quantity of trees in Amazonia. Since well developed models for single neurons already exist (Bower and Beeman, 2003; Carnevale and Hines, 2006), with complex dendritic morphology along with myriod of equations and parameters (Dayan and Abbott, 2001), it is not plain which model can generally satisfy all factors [2].

To measure the functionality of a brain system, modeling of extensive networks, including thousands of neurons is needed. Computer models commonly utilized for simulations are based on dynamical systems given by ordinary differential equations (ODE). To illustrate the dynamics of firing patterns generated by a neuron, it is necessary to be contained variables of multiple time scales [3]. Most neurons have strong tendency to fire repetitively while receiving a constant stimulating current and are capable of a large degree of firing frequency as this stimulus is different and this electrical activity has illustrated temporal behavior is often chaotic by experiments [4].

Since the connection between neurons is only done by the action potentials at (electrical or chemical) synapses, the significant thing is to model the dynamics of these action potentials [2].

Starting from a complicated four-dimension $\mathrm{H}-\mathrm{H}-$ model, simpler models with less equations (de Schutter, 2010) can be performed. If we numerically integrate these ODEs with the Euler method with a large time step, we can arrive to maps with similar dynamical properties as the original systems (Rulkov, 2002; Izhikevich, 2004) [2].

To get a proper model of a neuron, it is not enough to put the right kind of currents together, tune the parameters, right resistance, rheobase, and firing frequencies to fire spikes. The model should be reproduced all the neurocomputational features of the neuron, from coexistence of resting and spiking states, spike latencies, subthreshold oscillations, to rebound spikes, among others [5].

Ion exchange membranes (IEMs) are for transportations among two solutions with different concentration and composition. (RM) the membrane resistance has a key role in electrodialytic process and can be influenced by internal ion concentration profile in both sides of membrane. It is known that $\mathrm{R}_{\mathrm{M}}$ increases with decreasing concentration. Also it can be explained by description of membrane as a structure of microchannels and microcavities [6]. 
From academic considerations and numerical simulations, neuronal current sources polarize adjacent cells. This polarization can generate an induced electric field adding to the electric field due to sources. This field has powerful frequencydependent properties. This system is corresponding to an equivalent $\mathrm{RC}$ circuit and always has low-pass filtering properties. Also, the attenuation of low frequencies depends on the geometry of fluids and membranes in the extracellular space [7].

It is useful to define the resistance of the extracellular space with spherical surface to infinity with

$$
\mathrm{R}=1 / 4 \pi \mathrm{r} \sigma^{\prime}
$$

where $\sigma=0.3 \mathrm{Sm}^{-1}$ is the conductivity of the extracellular space that $R \approx 50 \mathrm{k} \Omega$. With using the cell soma as an electrode, this quantity is extracellular impedance, this value illustrates an upper bound, because, with contribution of all parts of dendrites, the effectual radius would be larger and the resistance even lower. A global impedance is measured as

$Z_{\text {global }}=Z_{\text {electrode }}+Z_{\text {neurone }}+Z_{\text {extracellular. }}$ (2) The article includes no independent method at all for determining $Z_{\text {electrode }}$ or $Z_{\text {neurone, }}$ let alone to such accuracy. [8]

In a recent study, it is implied that a raise in the axial resistivity $R_{i}$ or the membrane capacitance $C_{m}$ will have a tendency to amplify the increase of spike-width with distance from soma and also generally reduce the spike amplitude, whereas the dependency on membrane capacitance may be different with soma distance. The most significant neuronal parameter assigning the spike amplitude can be the number of dendrites associated with soma and their diameters. These can lead to distances up to $\sim 100 \mu \mathrm{m}$ from the soma, the amplitude depends on the sum of the cross-sectional areas of the dendrites connecting to soma. This can clarify that close to the soma, the amplitude has in our idealized models been seen to be proportional to $\sim \mathrm{d}^{3 / 2}$, To measure the role of the passive electrical parameters and cell morphology in investigating spike shapes, the same somatic action potentials have been used for all cell models [9].

It is implied that the solution velocity and temperature played a crucial role in membrane transport properties. From the ohmic-resistancecorrected, it indicated that the flow velocity and temperature played different roles in changes of modulus and phase angle. The overall conductivity of IEMs from direct current measurement had a linear relationship with the pure membrane resistance from EIS measurement. These results are significant for performance of ion exchange membranes [10].

EIS can be considered to characterize the ion exchange membrane systems and sub layers. It can be shown that electrical double layer in a circuit is as a resistor. Also data with electrochemical impedance was considered beneficial for investigating membrane properties [11].

Based on experimental data, IEM resistance depends on the identity and properties of single-salt solutions. In fact, Membrane resistance are gone up with increasing hydration free energy of the counter-ion in bulk solution.[12]

It has been studied, the membrane resistance decreases considerably with increasing temperature that can be beneficial for fuel cells and membrane capacitive deionization in temperature range and low salt concentration [13].

In this research, referring to the previous studies that has investigated effective factors on membrane current and resistance, the influence of resistance is evaluated on the shape, amplitude of neuron spikes. Indeed, none of them has been used non-linear changes of resistance via time.

\section{Theoretical basis}

\subsection{Hodgkin -Huxley model}

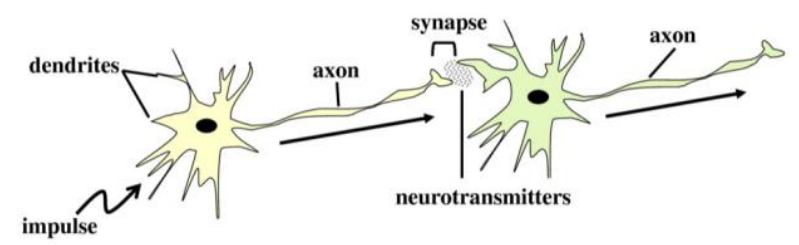

Fig. 1. anatomy of neuron with connection to another one through synapses

The Journal of physiology in 1952 would change the relationship between mathematics and physiology. Alan Lloyd Hodgkin and Andrew Huxley with a succession of five papers revolving around the nonlinear ordinary differential equations that can model action potentials to transfer through an axon. To illustrate, anatomy of neurons are depicted in Fig. 1. In fact, Neurons get electrical signals via dendrites. From there, the signal is transferred through the body and sent along the axon to the next one via action potentials as an explosion of depolarizing current. For firing an action potential (spikes), the depolarization must reach a minimum threshold voltage. At times, rheobase (current threshold) is determined to find time that spike is being produced. 
As it can be seen in Fig. 2. The $\mathrm{K}^{+}$travel due to both the $\mathrm{K}^{+}$concentration gradient and the electrical potential across the membrane. In in (a) For a cell with $\mathrm{K}^{+}$as a traveller, the concentration gradient of $\mathrm{K}^{+}$push $\mathrm{K}^{+}$ions out of the cell. In (b) The efflux of $\mathrm{K}^{+}$excess causes positive charge on the outside and a negative charge on the inside. At steady state, the electrical and chemical driving forces are equal and opposite.

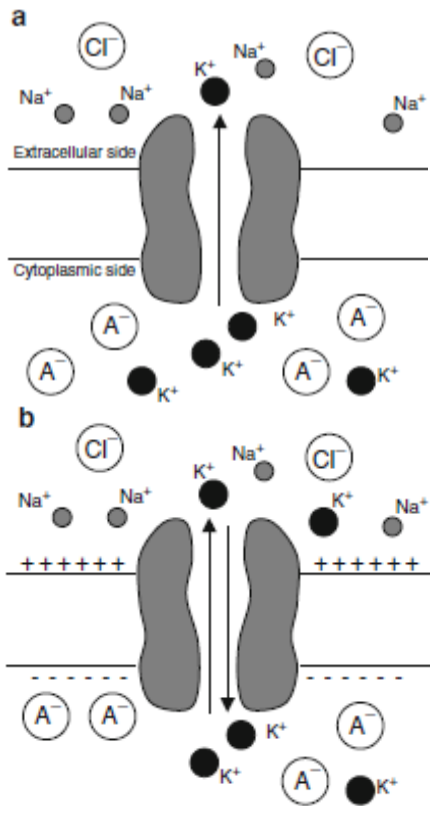

Fig. 2. The $\mathrm{K}^{+}$flux a) moving of $\mathrm{K}^{+}$due to concentration gradient $\mathrm{b}$ ) excess of positive charge on the outside and an excess of negative charge on the inside [14].

\subsection{Izhikevich model}

The value of the four parameters $a, b, c$, and d used in the Izhikevich model determines the spiking and bursting behavior of the known types of cortical neurons. The time evolution of the membrane potential $\mathrm{v}$ is described by the Differential equations:

\subsection{Equations}

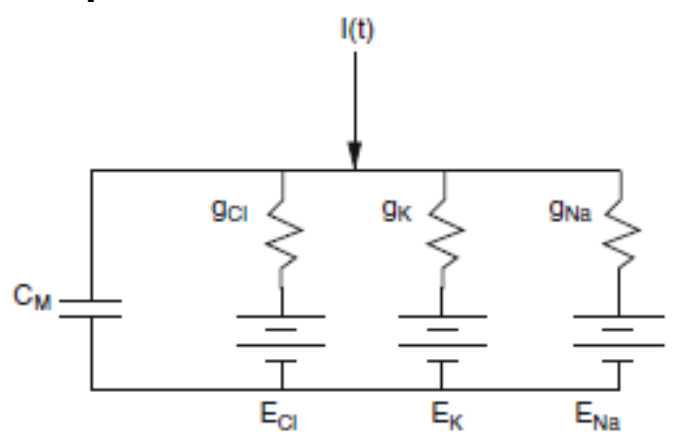

Fig. 3. equivalent circuit for a membrane with three channels

Fig. 3 illustrate an equivalent circuit with three parallel conductances and a current source, I ( $t)$. As a matter of fact, The current source is not as current per unit area, so we should divide $\mathrm{I}(\mathrm{t})$ by the total surface area of the neuron, A. It then follows that

$$
\begin{aligned}
& c_{M}\left(d V_{M} / d t\right)=-g_{C l}\left(V_{M}-E_{C l}\right)-g_{K}\left(V_{M}-E_{K}\right)-g_{N a}\left(V_{M}-\right. \\
& \left.\mathrm{E}_{\mathrm{Na}}\right)+\mathrm{I}(\mathrm{t}) / \mathrm{A} \\
& \left.\mathrm{c}_{\mathrm{M}}\left(\mathrm{dV} \mathrm{V}_{\mathrm{M}} / \mathrm{dt}\right)=-\left(\left(\mathrm{V}_{\mathrm{M}}-\mathrm{E}_{\mathrm{R}}\right) / \mathrm{r}_{\mathrm{M}}\right)\right)+\mathrm{I}(\mathrm{t}) / \mathrm{A} \\
& E_{R}=\left(g_{C l} E_{C l}+g_{K} E_{K}+g_{N a} E_{N a}\right) r_{M}
\end{aligned}
$$

Where $\mathrm{C}_{\mathrm{M}}$ is membrane capacitive, $\mathrm{V}_{\mathrm{M}}$ denotes membrane potential and $\mathrm{E}_{\mathrm{Na}}, \mathrm{E}_{\mathrm{K}}$, and $\mathrm{E}_{\mathrm{Cl}}$ are the Nernst equilibrium potentials where $\mathrm{V}^{*}=\mathrm{dV} / \mathrm{dt}$ is the derivative of the voltage with respect to time $t$. That arises because it takes time to charge the membrane. Also $V_{M}-E_{R}$ is driving force. and

$$
\mathrm{r}_{\mathrm{M}}=1 /\left(\mathrm{g}_{\mathrm{Cl}}+\mathrm{g}_{\mathrm{K}}+\mathrm{g}_{\mathrm{Na}}\right)
$$

That $r_{M}$ is the specific membrane resistance. These parameters, provided in the original Hodgkin and Huxley paper that the membrane potential shifted by approximately $65 \mathrm{mV}$, so that the resting potential is at $\mathrm{V} \approx 0$. Hodgkin and Huxley considered that for the sake of convenience, but has lot of confusion during years. The shifted Nernst equilibrium potentials are

$$
\begin{aligned}
& \mathrm{E}_{\mathrm{K}}=-12 \mathrm{mV} ; \\
& \mathrm{E}_{\mathrm{Na}}=120 \mathrm{mV} ; \\
& \mathrm{E}_{\mathrm{L}}=10.6 \mathrm{mV} ;
\end{aligned}
$$

Typical values of maximal conductance are

$$
\begin{aligned}
& \overline{\mathrm{g}}_{\mathrm{K}}=36 \mathrm{mS} / \mathrm{cm}^{2} ; \\
& \overline{\mathrm{g}}_{\mathrm{Na}}=120 \mathrm{mS} / \mathrm{cm}^{2} ; \\
& \mathrm{g}_{\mathrm{L}}=0.3 \mathrm{mS} / \mathrm{cm}^{2} ;
\end{aligned}
$$

$\mathrm{C}=1 \mu \mathrm{F} / \mathrm{cm}^{2}$ is the membrane capacitance and $\mathrm{I}=0$ $\mu \mathrm{A} / \mathrm{cm}^{2}$ is the applied current. The functions $\alpha(\mathrm{V})$ and $\beta(\mathrm{V})$ describe the transition rates between open and closed states of the channels.

As $\mathrm{V}_{\mathrm{M}}$ increases, the membrane potential difference begins to drive current across the membrane resistance, resulting in less current across the capacitor. Eventually, the membrane potential reaches a value where all the membrane current flows through the resistor. This value is given by $V_{M}$ $=\mathrm{I}_{0} \mathrm{R}_{\mathrm{INP}}$.

If there is no membrane capacitance, then $V_{M}$ satisfies

$$
V_{M}(t)=r_{M} I_{M}(t)
$$


Hodgkin and Huxley labeled the probability of a gate being in the permissive state as the name of the respective gate. To conform to this method, $\mathrm{p}_{\mathrm{i}}$ is exchanged for the name of the gates $-m, n$, or $h$. Each channel is defined to have a specific amount of these gates. For example, the sodium channel has three $\mathrm{m}$ gates and one $\mathrm{h}$ gate. [14] Using this notation and the above theory, the ionic currents can be summarized by,

$$
\begin{aligned}
& I_{\text {ion }}=\bar{g}_{N a} m^{3} h\left(V_{m}-E_{N a}\right)-\bar{g}_{k} n^{4}\left(V_{m}-E_{k}\right)-\bar{g}_{1}\left(V m-E_{1}\right) \\
& d n / d t=\alpha_{n}(V)(1-n)-\beta_{n}(V) n \\
& d m / d t=\alpha_{m}(V)(1-m)-\beta_{m}(V) m \\
& d h / d t=\alpha_{h}(V)(1-h)-\beta_{h}(V) h
\end{aligned}
$$$$
\mathrm{d} v / \mathrm{dt}=\left(1 / \mathrm{C}_{\mathrm{m}}\right) \cdot\left[\mathrm{I}-\mathrm{g}_{\mathrm{Na}} \mathrm{m}^{3} \mathrm{~h}\left(v-\mathrm{E}_{\mathrm{Na}}\right)-\mathrm{g}_{\mathrm{K}} \mathrm{n}^{4}\left(v-\mathrm{E}_{\mathrm{k}}\right)-\right.
$$$$
g_{1}\left(v-E_{l}\right)
$$

$\mathrm{dn} / \mathrm{dt}=\alpha_{\mathrm{n}}(v)(1-\mathrm{n})-\beta_{\mathrm{n}}(v) \mathrm{n}$

$\mathrm{dm} / \mathrm{dt}=\alpha_{\mathrm{m}}(v)(1-\mathrm{m})-\beta_{\mathrm{m}}(v) \mathrm{m}$

$\mathrm{dh} / \mathrm{dt}=\alpha_{\mathrm{h}}(v)(1-\mathrm{h})-\beta_{\mathrm{h}}(v) \mathrm{h}$

Where,

$$
\begin{aligned}
& \alpha_{\mathrm{n}}=[0.01(v+50) /(1-\exp (-(v+50) / 10)] \\
& \alpha_{\mathrm{m}}=[0.1(v+35) /(1-\exp (-(v+35) / 10)] \\
& \alpha_{\mathrm{h}}=0.07 \exp (-0.05(v+60)) \\
& \beta_{\mathrm{n}}=0.125 \exp [-(v+60) / 80] \\
& \beta_{\mathrm{m}}=4.0 \exp (-0.0556(v+60)) \\
& \beta \mathrm{h}=1 /[1+\exp (-0.1(v+30))]
\end{aligned}
$$

$$
\mathrm{CV}=\mathrm{I}-\mathrm{g}_{\text {inp }}(\mathrm{V}-\mathrm{Vrest}),
$$

Where

$$
\mathrm{g}_{\text {inp }}=\mathrm{g}_{\mathrm{Na}}+\mathrm{g}_{\mathrm{Ca}}+\mathrm{g}_{\mathrm{K}}+\mathrm{g}_{\mathrm{Cl}}
$$

Resting membrane potential is derived as follows:

$$
\begin{gathered}
\mathrm{V}_{\text {rest }}=\left[\left(g_{\mathrm{Na}} \mathrm{E}_{\mathrm{Na}}+\mathrm{g}_{\mathrm{Ca}} \mathrm{E}_{\mathrm{Ca}}+\mathrm{g}_{\mathrm{K}} \mathrm{E}_{\mathrm{K}}+\mathrm{g}_{\mathrm{Cl}} \mathrm{E}_{\mathrm{Cl}}\right) /\left(\mathrm{g}_{\mathrm{Na}}+\mathrm{g}_{\mathrm{Ca}}\right.\right. \\
\left.\left.+\mathrm{g}_{\mathrm{K}}+\mathrm{g}_{\mathrm{Cl}}\right)\right]
\end{gathered}
$$

In this study, resistance is defined as follows:
(25)

$$
\operatorname{deltaR}(\mathrm{i})=\sin (1 * \mathrm{t}(\mathrm{i})) * 0.0064 \text {; }
$$

Where

$$
\mathrm{R}(\mathrm{i}+1)=\operatorname{deltaR}(\mathrm{i})+\mathrm{R}(\mathrm{i}) \text {; }
$$

$$
\mathrm{R}(1)=0 \text { Initial resistance }
$$

And with conductance based on

$$
\begin{aligned}
& \overline{\mathrm{g}}_{\mathrm{K}}=36 \mathrm{mS} / \mathrm{cm}^{2} ; \\
& \overline{\mathrm{g}}_{\mathrm{Na}}=120 \mathrm{mS} / \mathrm{cm}^{2} ; \\
& \mathrm{g}_{\mathrm{L}}=0.3 \mathrm{mS} / \mathrm{cm}^{2} ;
\end{aligned}
$$

and equation (6) resistance is limited to 0.0064 .

The membrane potential in this research in defined based on the Ohm law as follows:

$$
\mathrm{V}(\mathrm{i})=\mathrm{R}(\mathrm{i}+1){ }^{*} \text { currentLevels; }
$$

\section{Results and discussion}

\subsection{Putting various currents in $\mathrm{H}-\mathrm{H}$ model}

The measurement were performed in three different current $(3,20,100)$ in the model. It can be seen in Fig. 4 by increasing the current, amplitude is decreasing along with the frequency is going up in accordance with the time scale from 0 to $100 \mathrm{~ms}$ in the simulated neuron.
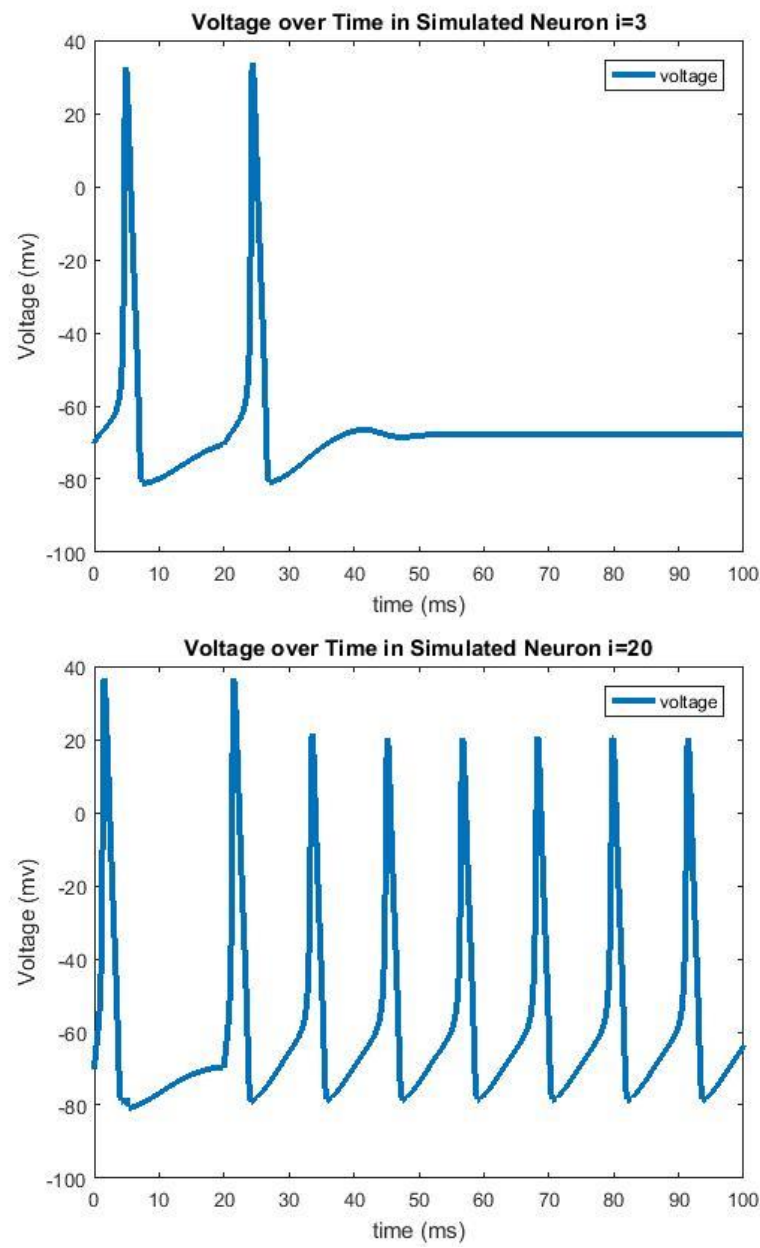


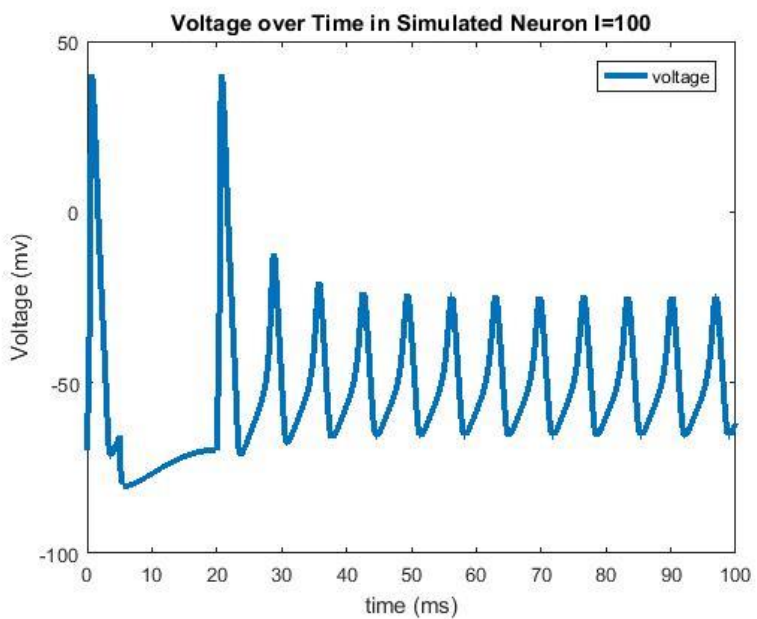

Fig. 4. Voltage over Time in various currents (attached MATLAB Code file named 3-1.m).

Regarding the Potassium and Sodium conductance in Fig. 5 in the higher current the number of spikes are escalating. In other words the frequency is growing also the initial value for the Potassium in $\mathrm{I}=100 \mathrm{~mA}$ has gone up meaning that Potassium are more sensitive to increase of stimulation.
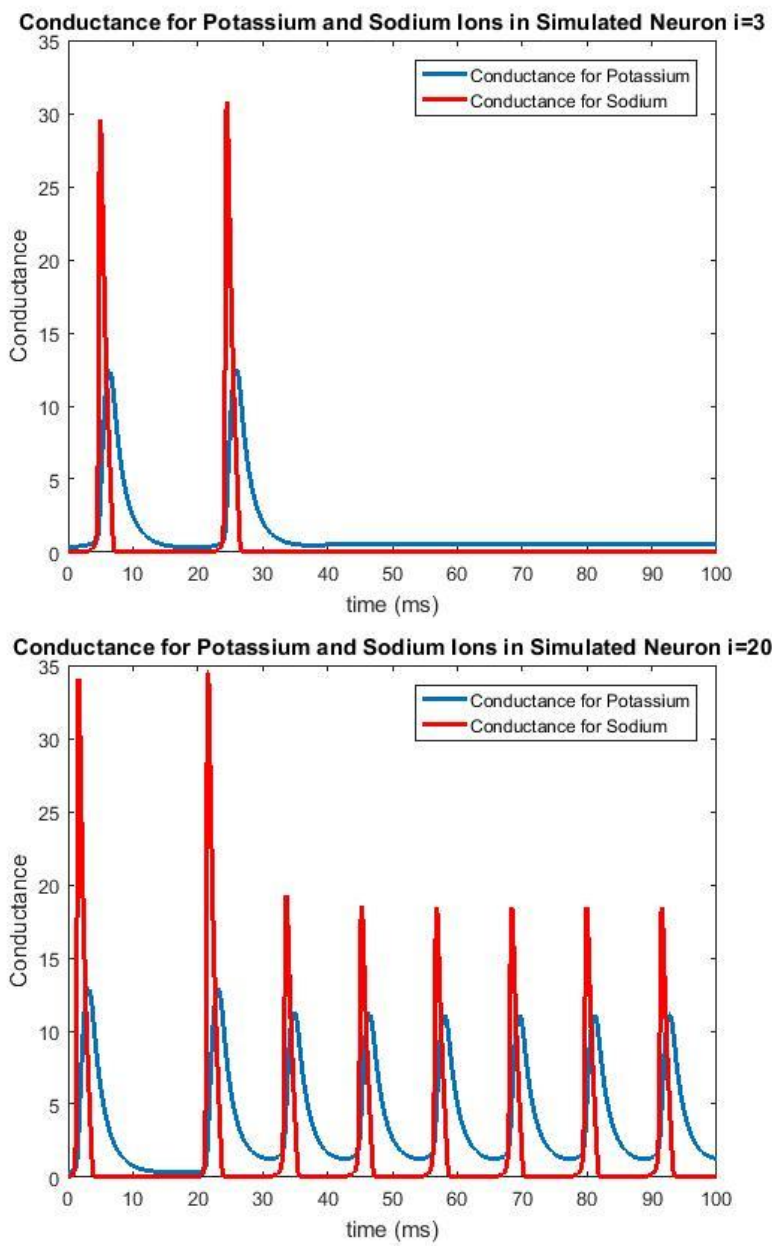

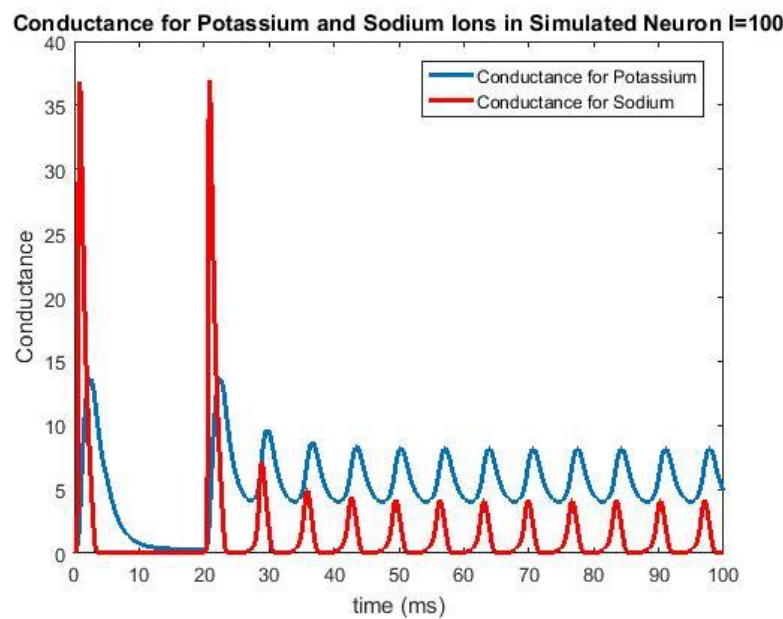

Fig. 5. Conductance for Potassium and Sodium Ions in various currents (attached MATLAB Code file named 3-1.m).

\subsection{Putting resistance factor in $\mathrm{H}-\mathrm{H}$ model}

In this study the resistance is assumed a valuable parameter that can influence the shape and width of spikes. So this factor is defined as a time dependent and based on the equation (25) is a linear value.

In Fig. 6 can be illustrated that the amplitude and frequency in various currents have the same trend. However the initial value and the peak are increasing.

The values of current for this section are referring to part 3-1. this pattern can be the results of Ohm law's nature.

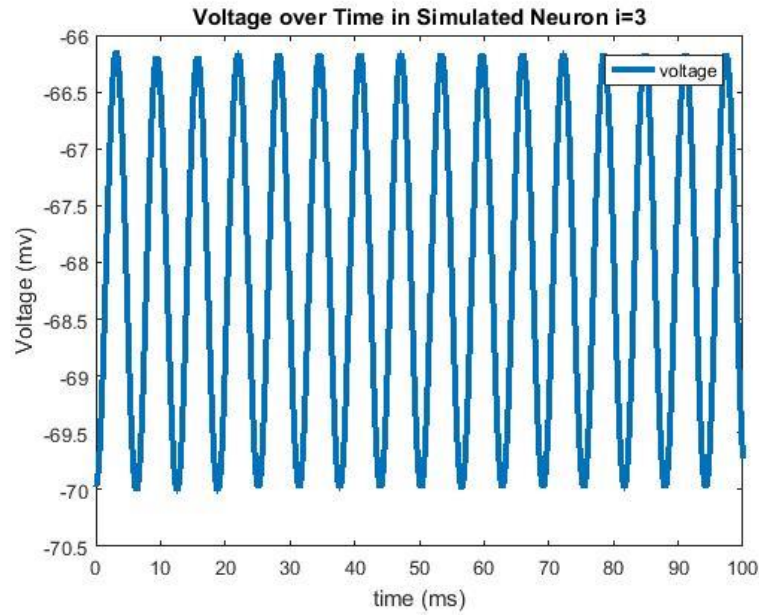



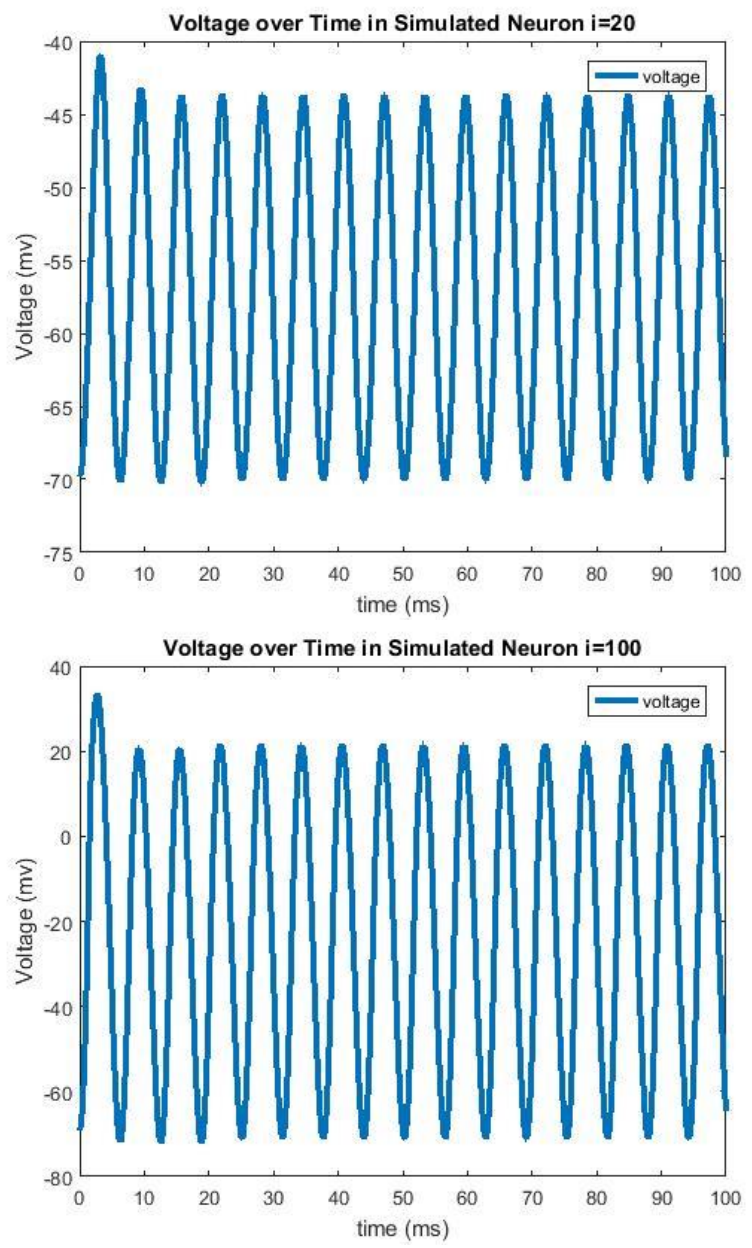

Fig. 6. Voltage over Time in various currents considering resistance as an effective factor (attached MATLAB Code file named 3-2.m).

According to Fig. 7 in contrast Fig. 5 with increasing the current due to the existence of resistance, amplitude is increasing and the Potassium and Sodium conductance patterns are approaching each other. Also it is found the latency for building of second spike is being shortened.
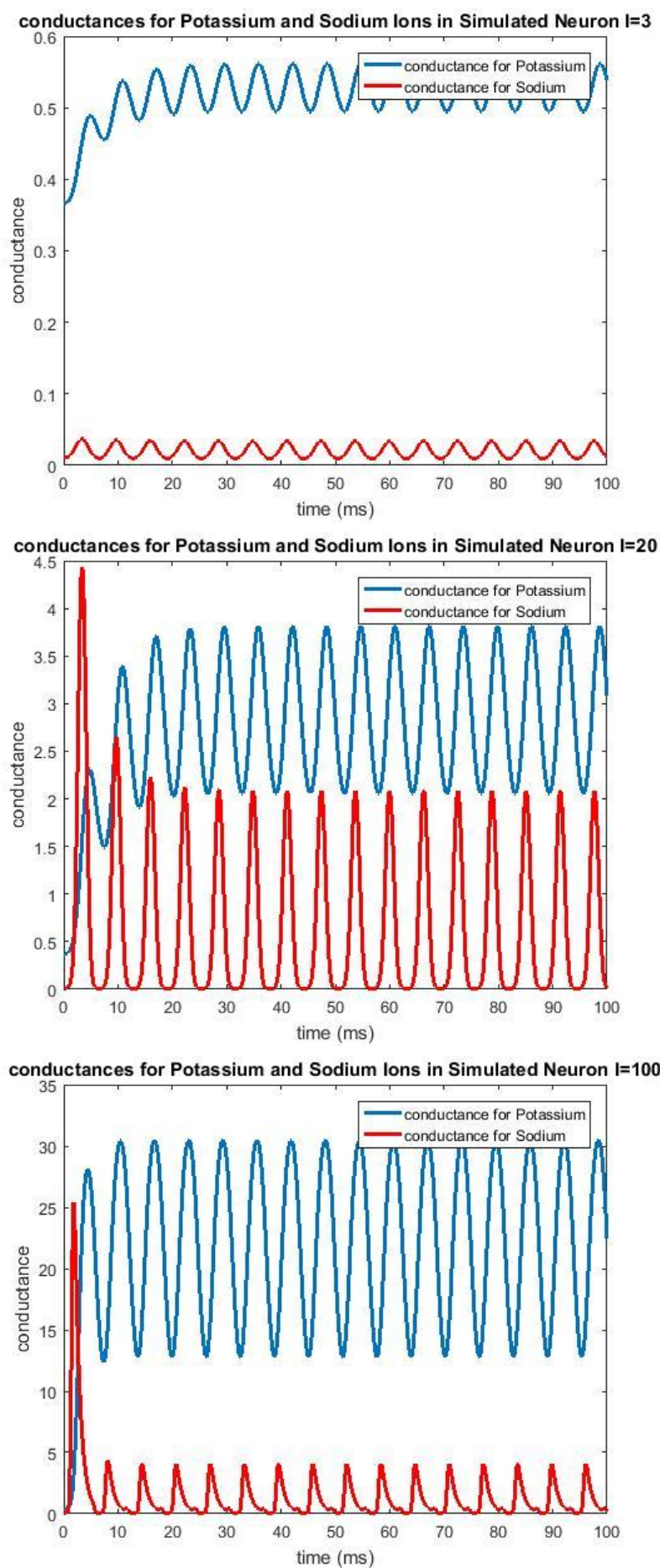

Fig. 7. Conductance for Potassium and Sodium Ions in various currents considering resistance as an effective factor (attached MATLAB Code file named 3-2.m).

\section{Conclusions}

It is assumed that the function of ion channels and interaction of current can produce heat and may change the impact of current in firing spikes that it is altering with passing time. 
In fact, morphology of dendrites, distance from main source and space and thickness of membrane and concentration of ions also can be investigated all together to be able to conclude the current threshold. Without considering resistance the number of spikes are increasing and the amplitude is going down; while with putting resistance in the codes the trend specially for the amplitude is different. The changes of resistance via potential can be derived from attached MATLAB Codes.

It is expected in the next studies the proper formula for membrane resistance, depending on time and temperature due to various factors including ion channels functions and their noise is defined. It can be beneficial to find more proper current and potential threshold result in describing helpful disease patterns. In the next article, two models will be compared in terms of time effect on changing resistance with each other.

\section{References:}

[1] Naureen Ghani, "Izhikevich Model of Cortical SpikingNeurons",

http://www.columbia.edu/cu/appliedneuroshp/Sprin g2018/Spring18SHPAppliedNeuroLec5.pdf,

February 24, 2018.

[2] M. Girardi-Schappo, M. H. R. Tragtenberg, O. Kinouchi, "A Brief History of Excitable Map-Based Neurons and Neural Networks", Journal of Neuroscience Methods, March 2013.

[3] N. F. Rulkov, I. Timofeev, M. Bazhenov, "Oscillations in Large-Scale Cortical Networks: Map-Based Model", Journal of Computational Neuroscience 17, 203-223, 2004

[4] John A.Connor, David Walter, and Russell Mckown, "Neural Repetitive Firing, Modifications of the Hodgkin-Huxley Axon Suggested by experimental results from crustacean axons", Biophysical Journal, Volume 18, 1977

[5] E. Izhikevich, "Simple model of spiking neurons," IEEE Transactions on Neural Networks, Vol. 14, No. 6, 1569-1572, Nov 2003.

[6] A.H. Galama, D.A. Vermaas, J. Veerman, M. Saakes, H.H.M. Rijnaarts, J.W. Post, K. Nijmeijer, "Membrane resistance: The effect of salinity gradients over a cation exchange membrane", Journal of Membrane Science, 467(2014) 279-291.

[7] C. Bédard, H. Kröger, and A. Destexhe, "Model of low-pass filtering of local field potentials in brain tissue", Physical Review, E 73, 051911 (2006).

[8] A. Plested, "Analysis of Claims that the Brain Extracellular Impedance Is High and Non-resistive", Biophysical Journal, 113, 1636-1638, October 3, 2017
[9] Klas H. Pettersen and Gaute T. Einevoll, "Amplitude Variability and Extracellular Low-Pass Filtering of Neuronal Spikes", Biophysical Journal, Volume 94 February 2008 784-802

[10] Wenjuan Zhang, Panpan Wang, Jun Ma, Zhenghui Wang, Huiling Liu, "Investigations on electrochemical properties of membrane systems in ion-exchange membrane transport processes by electrochemical impedance spectroscopy and direct current measurements", Electrochimica, Acta 216 (2016) 110-119

[11] Wenjuan Zhang, Jun Ma, Panpan Wang, Zhenghui Wang, Fengmei Shi, Huiling Liu, “ Investigations on the interfacial capacitance and the diffusion boundary layer thickness of ion exchange membrane using electrochemical impedance spectroscopy", Journal of Membrane Science, 502 (2016) 37-47

[12] Shan Zhu, Ryan S. Kingsbury, Douglas F. Call, Orlando Coronell, "Impact of solution composition on the resistance of ion exchange Membranes", Journal of Membrane Science, 554 (2018) 39-47

[13] Piotr Długoł eckia,b, Piotr Ogonowskib, Sybrand J. Metzb, Michel Saakesb, Kitty Nijmeijera, Matthias Wesslinga, "On the resistances of membrane, diffusion boundary layer and double layer in ion exchange membrane transport", Journal of Membrane Science, 349 (2010) 369-379

[14] R. Siciliano, "The Hodgkin - Huxley Model; Its Extensions, Analysis and Numerics", McGill Univ., Dept. Math. and Statist., Montreal, Canada, 2012.

Contribution of Individual Authors to the Creation of a Scientific Article (Ghostwriting Policy)

The authors equally contributed in the present research, at all stages from the formulation of the problem to the final findings and solution.

Sources of Funding for Research Presented in a Scientific Article or Scientific Article Itself

This paper research was supported by a grant from the Natural Sciences and Engineering Research Council (NSERC) of Canada.

\section{Conflict of Interest}

The authors have no conflicts of interest to declare that are relevant to the content of this article.

Creative Commons Attribution License 4.0 (Attribution 4.0 International, CC BY 4.0)

This article is published under the terms of the Creative Commons Attribution License 4.0 https://creativecommons.org/licenses/by/4.0/deed.en 